J. Appl. Numer. Optim. 1 (2019), No. 1, pp. 91-101

Available online at http://jano.biemdas.com

https://doi.org/10.23952/jano.1.2019.1.08

\title{
A DESCENT-LIKE METHOD FOR FIXED POINTS AND SPLIT CONCLUSION PROBLEMS
}

\author{
MEIJUAN SHANG \\ College of Science, Shijiazhuang University, Shijiazhuang 050035, China
}

\begin{abstract}
In this paper, a descent-like method is introduced for solving a fixed point problem of a strict pseudocontraction and a split variational inclusion problem. A strong convergence theorem of common solutions is established in the framework of Hilbert spaces without any compact assumptions on any mapping.
\end{abstract}

Keywords. Iterative algorithm; Monotone operator; Split inclusion problem; Variational inequality.

2010 Mathematics Subject Classification. 47H05, 47H09, 47J20, 65K15.

\section{INTRODUCTION-PRELIMINARIES}

Let $H_{1}$ and $H_{2}$ be two real Hilbert spaces endowed with inner products and induced norms denoted by $\langle\cdot, \cdot\rangle$ and $\|\cdot\|$, respectively, while $H$ refers to as any of these spaces.

Let $M: H \rightarrow 2^{H}$ be a monotone mapping. $M$ is said to be monotone iff, for all $x, y \in H_{1}, u \in M x$ and $v \in M y$

$$
\langle x-y, u-v\rangle \geq 0 .
$$

It is said to be maximal iff the $\operatorname{Graph}(M)$ is not properly contained in the graph of any other monotone mapping. It is known that the graph of maximal monotone operators is weakly-strongly closed [1]. It is also known that a monotone mapping $M$ is maximal iff for $(x, u) \in H \times H,\langle x-y, u-v\rangle \geq 0$, for every $(y, v) \in \operatorname{Graph}(M)$ implies that $u \in M(x)$. Let $r$ be some positive real number. The resolvent mapping, $\operatorname{Res}_{r}^{M}: H \rightarrow H$ associated with mapping $M$ and number $r$, is defined by

$$
\operatorname{Res}_{r}^{M} x=(I+r M)^{-1}(x), \forall x \in H,
$$

where $I$ stands for identity operator on $H$. If $M$ is the subdifferential of proper convex lower semicontinuous functions, then the resolvent operator is the called the proximity operator. It is known that $\operatorname{Fix}\left(\operatorname{Res}_{r}^{M}\right)=M^{-1}(0)$, where $F i x\left(\operatorname{Res}_{r}^{M}\right)$ stand for the fixed-point set of $\operatorname{Res}_{r}^{M}$. The resolve operator plays an important role in many convex optimization problem and many authors studied zero points of maximal monotone operators via resolvent techniques; see, e.g., [2, 3, 4, 5, 6, 7] and the references therein.

Let $A: H_{1} \rightarrow H_{2}$ be a bounded linear operator. Let $M$ and $N$ be two maximal monotone operators on $H_{1}$ and $H_{2}$, respectively. In this paper, we study the following split variational inclusion problem: Find $x^{*} \in H_{1}$ such that

$$
0 \in M\left(x^{*}\right)
$$

E-mail address: meijuanshang@163.com

Received December 4, 2018; Accepted March 23, 2019. 
and

$$
y^{*}=A x^{*} \in H_{2} \quad \text { solves } \quad 0 \in N\left(y^{*}\right) .
$$

In this paper, we use $\operatorname{SF} P(M, N)$ to denote the solution set of the split variational inclusion problem. In 1994, Censor and Elfving [8] first introduced a split feasibility problem in finite-dimensional Hilbert spaces for modeling inverse problems which arise from phase retrievals and in medical image reconstruction. It has been found that the split variational inclusion problem can be used in various disciplines such as image restoration, computer tomograph and radiation therapy treatment planning $[9,10,11]$. Recently, the above split variational inclusion problem has been studied based on Mann-like methods by many authors; see $[12,13,14,15]$ and the references therein.

Let $T$ be a mapping on $H$. Recall that $T$ is said to be nonexpansive iff

$$
\|T x-T y\| \leq\|x-y\|, \quad \forall x, y \in H .
$$

$T$ is said to be quasi-nonexpansive iff $\operatorname{Fix}(T) \neq \emptyset$ and

$$
\|x-T y\| \leq\|x-y\|, \quad \forall x \in \operatorname{Fix}(T), y \in H .
$$

It is known that every nonexpansive mapping satisfies the following properties

$$
\langle T x-T y,(y-T y)-(x-T x)\rangle \leq \frac{1}{2}\|(x-T x)-(y-T y)\|^{2}, \quad \forall x, y \in H .
$$

In particular, every quasi-nonexpansive mapping satisfies the following properties

$$
\langle x-T y,(y-T y)\rangle \leq \frac{1}{2}\|y-T y\|^{2}, \quad \forall x \in \operatorname{Fix}(T), y \in H .
$$

Recall that $T$ is said to be firmly nonexpansive iff

$$
\|T x-T y\|^{2} \leq\langle T x-T y, x-y\rangle, \quad \forall x, y \in H .
$$

It is known that the resolvents of maximal monotone operators is firmly nonexpansive. $T$ is said to be firmly quasi-nonexpansive iff $\operatorname{Fix}(T) \neq \emptyset$ and

$$
\|x-T y\|^{2} \leq\langle x-T y, x-y\rangle, \quad \forall x \in F i x(T), y \in H .
$$

Recall that $T$ is said to be strictly pseudocontractive iff there is a real number $\kappa \in[0,1)$ such that

$$
\|T x-T y\|^{2} \leq\|x-y\|+\kappa\|(I-T) x-(I-T)\|^{2}, \quad \forall x, y \in D .
$$

The class of strictly pseudocontractive mappings was first introduced and studied by Browder and Petryshy [16] in the framework of Hilbert spaces. Since then, many authors have studied fixed points of strictly pseudocontractive mappings via different methods and techniques; see $[17,18,19]$ and the references therein.

In this paper, we investigate the split variational inclusion problem which involving a strict pseudocontractive mapping via a fixed-point method in an infinite dimensional Hilbert spaces. Strong convergence theorems are established without any compact assumptions on mappings.

The following lemmas are essential for our main results.

Lemma 1.1. [16] Let $H$ be a real Hilbert space. Let $T$ be a $\kappa$-strict pseudocontraction with fixed points and let $\left\{\beta_{n}\right\}$ be a sequence in $(0,1)$. Define a mapping $S$ by

$$
T_{n} x=\left(1-\beta_{n}\right) T x+\beta_{n} x,
$$


$\forall x \in H$. If $\beta \in[\kappa, 1)$, then $T_{n}$ is nonexpansive and Fix $\left(T_{n}\right)=F i x(T)$ for each $n \geq 1$. If $\left\{x_{n}\right\}$ converges weakly to some point $x^{*}$ and $x_{n}-T x_{n} \rightarrow 0$ as $n \rightarrow \infty$, then $x^{*} \in F i x(T)$, that is, $I-T$, where $I$ is the identity mapping, is demiclosed at zero.

Lemma 1.2. [20] Let $H$ be a Hilbert space and let $F$ be an $\eta$-strongly monotone, $\mathscr{L}$-Lipschitz continuous mapping on $H$. Define a mapping $T^{\alpha}: H \rightarrow H$ by $T^{\alpha} x=(I-\mu \alpha F) x, \forall x \in H$, where $\alpha$ is a real number in $(0,1)$. If $0<\mu \in\left(0, \frac{2 \eta}{\mathscr{L}^{2}}\right)$, then $T^{\alpha}$ is a contraction, that is,

$$
\left\|T^{\alpha} x-T^{\alpha} y\right\| \leq(1-\alpha \tau)\|x-y\|,
$$

$\forall x, y \in H$, where $\tau=1-\sqrt{1-\mu\left(2 \eta-\mu \mathscr{L}^{2}\right)} \in(0,1]$.

Lemma 1.3. [21] Let $H$ be a real Hilbert space and let $M$ be a maximal operator. For $\lambda>0$ and $\mu>0$, we have

$$
(I+\mu M)^{-1}\left(\frac{\mu}{\lambda} x+\left(1-\frac{\mu}{\lambda}\right)(I+\lambda M)^{-1} x\right)=(I+\lambda M)^{-1} x, \forall x \in H .
$$

Lemma 1.4. [22] Let $H$ be a real Hilbert space. Let $\left\{x_{n}\right\}$ and $\left\{y_{n}\right\}$ be bounded sequences in H. Let $\left\{\sigma_{n}\right\}$ be a sequence in $(0,1)$ such that

$$
0<\liminf _{n \rightarrow \infty} \sigma_{n} \leq \limsup _{n \rightarrow \infty} \sigma_{n}<1 .
$$

Assume that $x_{n+1}=\sigma_{n} y_{n}+\left(1-\sigma_{n}\right) x_{n}$ and

$$
\limsup _{n \rightarrow \infty}\left(\left\|y_{n+1}-y_{n}\right\|-\left\|x_{n+1}-x_{n}\right\|\right) \leq 0 .
$$

Then $\lim _{n \rightarrow \infty}\left\|y_{n}-x_{n}\right\|=0$.

Lemma 1.5. [23] Let $\left\{\alpha_{n}\right\},\left\{\beta_{n}\right\}$ and $\left\{\gamma_{n}\right\}$ be sequences of real numbers such that $\alpha_{n} \in[0,1], \sum_{n=1}^{\infty} \alpha_{n}=$ $\infty$, limsup $\operatorname{su}_{n \rightarrow \infty} \beta_{n} \leq 0$ and $\sum_{n=1}^{\infty} \gamma_{n}<\infty$ Let $\left\{\lambda_{n}\right\}$ be a sequence of nonnegative real numbers such that

$$
\lambda_{n+1} \leq\left(1-\alpha_{n}\right) \lambda_{n}+\alpha_{n} \beta_{n}+\gamma_{n}
$$

Then $\lim _{n \rightarrow \infty} \lambda_{n}=0$.

\section{MAIN RESULTS}

Theorem 2.1. Let $H_{1}$ and $H_{2}$ be two real Hilbert spaces. Let $T$ be a strictly pseudocontractive mapping with coefficient $\kappa \in[0,1)$ on $H_{1}$ with fixed points. Let $A: H_{1} \rightarrow H_{2}$ be a bounded linear operator and let $A^{*}$ be the adjoint operator of $A$. Let $F: H_{1} \rightarrow H_{1}$ be a $\mathscr{L}$-Lipschitz continuous and $\tau$-strongly monotone mapping. Let $M$ be a maximal monotone mapping on $H_{1}$ and let $N$ be a maximal monotone mapping on $H_{2}$. Assume that $\operatorname{SFP}(M, N) \cap \operatorname{Fix}(T) \neq \emptyset$. Let $\left\{x_{n}\right\}$ be a sequence generated by the following process:

$$
\left\{\begin{array}{l}
x_{1} \in H_{1}, \\
y_{n}=\operatorname{Res}_{r_{n}}^{M}\left(x_{n}+\gamma A^{*}\left(\operatorname{Res}_{s_{n}}^{N}-I\right) A x_{n}\right), \\
z_{n}=\left(1-\beta_{n}\right) T y_{n}+\beta_{n} y_{n}, \\
x_{n+1}=\left(1-\gamma_{n}\right) x_{n}+\gamma_{n}\left(I-\mu \alpha_{n} F\right) z_{n}, \quad n \geq 1,
\end{array}\right.
$$

where $\left\{r_{n}\right\}$ and $\left\{s_{n}\right\}$ are two positive real number sequences, $\left\{\alpha_{n}\right\},\left\{\beta_{n}\right\}$ and $\left\{\gamma_{n}\right\}$ are sequences in $(0,1), \mu$ and $\gamma$ are two positive real numbers. Assume that $\lim _{n \rightarrow \infty} \alpha_{n}=0, \sum_{n=1}^{\infty} \alpha_{n}=\infty,\left\{\beta_{n}\right\}$ is in 
$[\kappa, \beta)$, where $\kappa \leq \beta<1$, such that $\lim _{n \rightarrow \infty}\left|\beta_{n+1}-\beta_{n}\right|=0,\left\{\gamma_{n}\right\}$ is in $\left[\rho, \rho^{\prime}\right]$, where $\rho$ and $\rho^{\prime}$ are $t$ wo real numbers in $(0,1)$, such that $\lim _{n \rightarrow \infty}\left|\gamma_{n+1}-\gamma_{n}\right|=0, \liminf _{n \rightarrow \infty} r_{n}>0, \lim _{n \rightarrow \infty}\left|r_{n+1}-r_{n}\right|<\infty$, $\liminf _{n \rightarrow \infty} s_{n}>0, \lim _{n \rightarrow \infty}\left|s_{n+1}-s_{n}\right|<\infty, \mu \in\left(0, \frac{2 \tau}{\mathscr{L}^{2}}\right)$, and $\gamma \in\left(0, \frac{1}{\|A\|^{2}}\right)$. Then $\left\{x_{n}\right\}$ converge strongly to $\bar{x} \in \operatorname{SFP}(M, N) \cap F i x(T)$, which is the unique solution of the following variational inequality

$$
\langle\bar{x}-y, F \bar{x}\rangle \leq 0, \quad \forall y \in \operatorname{SFP}(M, N) \cap F i x(T) .
$$

Proof. Set

$$
T_{n}=\left(1-\beta_{n}\right) T+\beta_{n}, \quad \forall n \geq 1 .
$$

Since $\left\{\beta_{n}\right\}$ is in $[\kappa, 1)$, we obtain from Lemma 1.1 that $T_{n}$ is a nonexpansive mapping and $\operatorname{Fix}(T)=F\left(T_{n}\right)$ for each $n$. Note that $x \in \operatorname{SFP}(M, N) \cap F i x(T)$ iff $x=\operatorname{Res}_{r_{n}}^{M} x, A x=\operatorname{Res}_{s_{n}}^{N} A x$, and $x=T x$. Since both the resolvents are firmly nonexpansive, one has

$$
\begin{aligned}
\left\|y_{n}-x\right\|^{2}= & \left\|\operatorname{Res}_{r_{n}}^{M}\left(x_{n}+\gamma A^{*}\left(\operatorname{Res}_{s_{n}}^{N}-I\right) A x_{n}\right)-\operatorname{Res}_{r_{n}}^{M} x\right\|^{2} \\
\leq & \left\|\left(x_{n}-x\right)+\gamma A^{*}\left(\operatorname{Res}_{s_{n}}^{N}-I\right) A x_{n}\right\|^{2} \\
\leq & \left\|x_{n}-x\right\|^{2}+2 \gamma\left\langle A^{*}\left(\operatorname{Res}_{s_{n}}^{N}-I\right) A x_{n}, x_{n}-x\right\rangle+\gamma^{2}\|A\|^{2}\left\|\left(\operatorname{Res}_{s_{n}}^{N}-I\right) A x_{n}\right\|^{2} \\
\leq & \left\|x_{n}-x\right\|^{2}+2 \gamma\left\langle\left(\operatorname{Res}_{s_{n}}^{N}-I\right) A x_{n}, A\left(x_{n}-x\right)+\left(\operatorname{Res}_{s_{n}}^{N}-I\right) A x_{n}\right\rangle \\
& -2 \gamma\left\langle\left(\operatorname{Res}_{s_{n}}^{N}-I\right) A x_{n},\left(\operatorname{Res}_{s_{n}}^{N}-I\right) A x_{n}\right\rangle+\gamma^{2}\|A\|^{2}\left\|\left(\operatorname{Res}_{s_{n}}^{N}-I\right) A x_{n}\right\|^{2} .
\end{aligned}
$$

Note that $A x$ is a fixed point of $\operatorname{Res}_{s_{n}}^{N}$. Using (1.3), we see that

$$
\left\langle A\left(x_{n}-x\right)+\left(\operatorname{Res}_{s_{n}}^{N}-I\right) A x_{n},\left(\operatorname{Res}_{s_{n}}^{N}-I\right) A x_{n}\right\rangle \leq \frac{1}{2}\left\|\left(\operatorname{Res}_{s_{n}}^{N}-I\right) A x_{n}\right\|^{2}
$$

It follows that

$$
\left\|y_{n}-x\right\|^{2} \leq\left\|x_{n}-x_{\|}^{2}-\gamma\left(1-\gamma\|A\|^{2}\right)\right\|\left(\operatorname{Res}_{s_{n}}^{N}-I\right) A x_{n} \|^{2} .
$$

Since $\gamma \in\left(0, \frac{1}{\|A\|^{2}}\right)$, we have $\left\|y_{n}-x\right\| \leq\left\|x-x_{n}\right\|$. It follows from the nonexpansivity of $T_{n}$ that

$$
\left\|z_{n}-x\right\|=\left\|\left(1-\beta_{n}\right) T y_{n}+\beta_{n} y_{n}-x\right\|=\left\|T_{n} y_{n}-T_{n} x\right\| \leq\left\|y_{n}-x\right\| \leq\left\|x-x_{n}\right\| .
$$

Using Lemma 1.2, we have

$$
\begin{aligned}
\left\|x_{n+1}-x\right\| & \leq \gamma_{n}\left\|\left(I-\mu \alpha_{n} F\right) z_{n}-\left(I-\mu \alpha_{n} F\right) x-\mu \alpha_{n} F x\right\|+\left(1-\gamma_{n}\right)\left\|x_{n}-x\right\| \\
& \leq \gamma_{n}\left\|\left(I-\mu \alpha_{n} F\right) z_{n}-\left(I-\mu \alpha_{n} F\right) x\right\|+\mu \alpha_{n} \gamma_{n}\|F x\|+\left(1-\gamma_{n}\right)\left\|x_{n}-x\right\| \\
& \leq \gamma_{n}\left(1-\tau \alpha_{n}\right)\left\|z_{n}-x\right\|+\mu \alpha_{n} \gamma_{n}\|F x\|+\left(1-\gamma_{n}\right)\left\|x_{n}-x\right\| \\
& \leq\left(1-\gamma_{n} \tau \alpha_{n}\right)\left\|x_{n}-x\right\|+\alpha_{n} \mu \gamma_{n}\|F x\| \\
& \leq \max \left\{\left\|x_{n}-x\right\|, \frac{\mu}{\tau}\|F x\|\right\} .
\end{aligned}
$$


This implies that $\left\{x_{n}\right\}$ is a bounded vector sequence, so are $\left\{y_{n}\right\}$ and $\left\{z_{n}\right\}$. Using Lemma 1.3, we find that

$$
\begin{aligned}
& \left\|\left(x_{n+1}+\gamma A^{*}\left(\operatorname{Res}_{s_{n+1}}^{N}-I\right) A x_{n+1}\right)-\left(x_{n}+\gamma A^{*}\left(\operatorname{Res}_{s_{n}}^{N}-I\right) A x_{n}\right)\right\| \\
& =\left\|x_{n+1}-x_{n}+\gamma A^{*}\left(A x_{n}-A x_{n+1}+\operatorname{Res}_{s_{n}}^{N} A x_{n+1}-\operatorname{Res}_{s_{n+1}}^{N} A x_{n}\right)\right\| \\
& \leq\left\|x_{n+1}-x_{n}-\gamma A^{*}\left(A x_{n+1}-A x_{n}\right)\right\|+\left\|\gamma A^{*}\left(\operatorname{Res}_{s_{n}}^{N} A x_{n}-\operatorname{Res}_{s_{n+1}}^{N} A x_{n+1}\right)\right\| \\
& \leq\left(1-\gamma\|A\|^{2}\right)\left\|x_{n+1}-x_{n}\right\|+\gamma\|A\|\left\|\operatorname{Res}_{s_{n}}^{N} A x_{n}-\operatorname{Res}_{s_{n}}^{N}\left(\frac{s_{n}}{s_{n+1}} A x_{n+1}+\left(1-\frac{s_{n}}{s_{n+1}}\right) \operatorname{Res}_{s_{n+1}}^{N} A x_{n+1}\right)\right\| \\
& \leq\left(1-\gamma\|A\|^{2}\right)\left\|x_{n+1}-x_{n}\right\|+\gamma\|A\|\left(\frac{\left|s_{n+1}-s_{n}\right|}{s_{n+1}}\left\|\operatorname{Res}_{s_{n+1}}^{N} A x_{n+1}-A x_{n+1}\right\|+\left\|A x_{n+1}-A x_{n}\right\|\right) \\
& \leq\left\|x_{n}-x_{n+1}\right\|+\frac{\left|s_{n+1}-s_{n}\right|}{s_{n+1}} \gamma\|A\|\left\|\operatorname{Res}_{s_{n+1}}^{N} A x_{n+1}-A x_{n+1}\right\|
\end{aligned}
$$

and

$$
\begin{aligned}
\| & \operatorname{Res}_{r_{n+1}}^{M}\left(x_{n+1}+\gamma A^{*}\left(\operatorname{Res}_{s_{n+1}}^{N}-I\right) A x_{n+1}\right)-\operatorname{Res}_{r_{n}}^{M}\left(x_{n+1}+\gamma A^{*}\left(\operatorname{Res}_{s_{n+1}}^{N}-I\right) A x_{n+1}\right) \| \\
= & \| \operatorname{Res}_{r_{n}}^{M}\left(\frac{r_{n}}{r_{n+1}}\left(x_{n+1}+\gamma A^{*}\left(\operatorname{Res}_{s_{n+1}}^{N}-I\right) A x_{n+1}\right)\right. \\
& \left.+\left(1-\frac{r_{n}}{r_{n+1}}\right) \operatorname{Res}_{r_{n+1}}^{M}\left(x_{n+1}+\gamma A^{*}\left(\operatorname{Res}_{s_{n+1}}^{N}-I\right) A x_{n+1}\right)\right) \\
& -\operatorname{Res}_{r_{n}}^{M}\left(x_{n+1}+\gamma A^{*}\left(\operatorname{Res}_{s_{n+1}}^{N}-I\right) A x_{n+1}\right) \| \\
\leq & \|\left(\frac{r_{n}}{r_{n+1}}\left(x_{n+1}+\gamma A^{*}\left(\operatorname{Res}_{s_{n+1}}^{N}-I\right) A x_{n+1}\right)\right. \\
& \left.+\left(1-\frac{r_{n}}{r_{n+1}}\right) \operatorname{Res}_{r_{n+1}}^{M}\left(x_{n+1}+\gamma A^{*}\left(\operatorname{Res}_{s_{n+1}}^{N}-I\right) A x_{n+1}\right)\right) \\
& -\left(x_{n+1}+\gamma A^{*}\left(\operatorname{Res}_{s_{n+1}}^{N}-I\right) A x_{n+1}\right) \| \\
\leq & \frac{\left|r_{n+1}-r_{n}\right|}{r_{n+1}} \|\left(x_{n+1}+\gamma A^{*}\left(\operatorname{Res}_{s_{n+1}}^{N}-I\right) A x_{n+1}\right) \\
& \left.-\operatorname{Res}_{r_{n+1}}^{M}\left(x_{n+1}+\gamma A^{*}\left(\operatorname{Res}_{s_{n+1}}^{N}-I\right) A x_{n+1}\right)\right) \| .
\end{aligned}
$$

On the other hand, one has

$$
\begin{aligned}
& \left\|y_{n+1}-y_{n}\right\| \\
& \leq\left\|\operatorname{Res}_{r_{n+1}}^{M}\left(x_{n+1}+\gamma A^{*}\left(\operatorname{Res}_{s_{n+1}}^{N}-I\right) A x_{n+1}\right)-\operatorname{Res}_{r_{n}}^{M}\left(x_{n+1}+\gamma A^{*}\left(\operatorname{Res}_{s_{n+1}}^{N}-I\right) A x_{n+1}\right)\right\| \\
& \quad+\left\|\operatorname{Res}_{r_{n}}^{M}\left(x_{n+1}+\gamma A^{*}\left(\operatorname{Res}_{s_{n+1}}^{N}-I\right) A x_{n+1}\right)-\operatorname{Res}_{r_{n}}^{M}\left(x_{n}+\gamma A^{*}\left(\operatorname{Res}_{s_{n}}^{N}-I\right) A x_{n}\right)\right\| \\
& \leq\left\|\operatorname{Res}_{r_{n+1}}^{M}\left(x_{n+1}+\gamma A^{*}\left(\operatorname{Res}_{s_{n+1}}^{N}-I\right) A x_{n+1}\right)-\operatorname{Res}_{r_{n}}^{M}\left(x_{n+1}+\gamma A^{*}\left(\operatorname{Res}_{s_{n+1}}^{N}-I\right) A x_{n+1}\right)\right\| \\
& \quad+\left\|\left(x_{n+1}+\gamma A^{*}\left(\operatorname{Res}_{s_{n+1}}^{N}-I\right) A x_{n+1}\right)-\left(x_{n}+\gamma A^{*}\left(\operatorname{Res}_{s_{n}}^{N}-I\right) A x_{n}\right)\right\| .
\end{aligned}
$$

Substituting (2.3) and (2.4) into (2.5) yields that

$$
\left\|y_{n+1}-y_{n}\right\| \leq\left\|x_{n}-x_{n+1}\right\|+M\left(\frac{\left|r_{n+1}-r_{n}\right|}{r_{n+1}}+\frac{\left|s_{n+1}-s_{n}\right|}{s_{n+1}}\right),
$$

were $M$ is an appropriate constant. Since $T_{n}$ is nonexpansive, one obtains from (2.6) that

$$
\begin{aligned}
\left\|z_{n+1}-z_{n}\right\| & \leq\left\|T_{n+1} y_{n+1}-T_{n+1} y_{n}\right\|+\left\|T_{n+1} y_{n}-T_{n} y_{n}\right\| \\
& \leq\left\|y_{n+1}-y_{n}\right\|+\left|\beta_{n+1}-\beta_{n}\right|\left\|T y_{n}-y_{n}\right\| \\
& \leq\left\|x_{n}-x_{n+1}\right\|+M\left(\frac{\left|r_{n+1}-r_{n}\right|}{r_{n+1}}+\frac{\left|s_{n+1}-s_{n}\right|}{s_{n+1}}\right)+\left|\beta_{n+1}-\beta_{n}\right|\left\|T y_{n}-y_{n}\right\| .
\end{aligned}
$$


Setting $\lambda_{n}=\left(I-\mu \alpha_{n} F\right) z_{n}$, one has

$$
\begin{aligned}
& \left\|\lambda_{n+1}-\lambda_{n}\right\| \\
& \leq\left\|\left(I-\mu \alpha_{n+1} F\right) z_{n+1}-\left(I-\mu \alpha_{n+1} F\right) z_{n}\right\|+\left\|\left(I-\mu \alpha_{n+1} F\right) z_{n}-\left(I-\mu \alpha_{n} F\right) z_{n}\right\| \\
& \leq\left(1-\tau \alpha_{n+1}\right)\left\|z_{n+1}-z_{n}\right\|+\mu\left|\alpha_{n+1}-\alpha_{n}\right|\left\|F z_{n}\right\| \\
& \leq\left(1-\tau \alpha_{n+1}\right)\left\|x_{n}-x_{n+1}\right\|+M\left(\frac{\left|r_{n+1}-r_{n}\right|}{r_{n+1}}+\frac{\left|s_{n+1}-s_{n}\right|}{s_{n+1}}\right) \\
& \quad+\left|\beta_{n+1}-\beta_{n}\right|\left\|T y_{n}-y_{n}\right\|+\mu\left|\alpha_{n+1}-\alpha_{n}\right|\left\|F z_{n}\right\| .
\end{aligned}
$$

This implies that

$$
\begin{aligned}
\left\|\lambda_{n+1}-\lambda_{n}\right\|-\left\|x_{n}-x_{n+1}\right\| \leq & \left|\beta_{n+1}-\beta_{n}\right||| T y_{n}-y_{n}\left\|+\mu\left|\alpha_{n+1}-\alpha_{n}\right||| F z_{n}\right\| \\
& +M\left(\frac{\left|r_{n+1}-r_{n}\right|}{r_{n+1}}+\frac{\left|s_{n+1}-s_{n}\right|}{s_{n+1}}\right) .
\end{aligned}
$$

Using the restrictions imposed on the control sequences, one finds that

$$
\limsup _{n \rightarrow \infty}\left(\left\|\lambda_{n+1}-\lambda_{n}\right\|-\left\|x_{n}-x_{n+1}\right\|\right) \leq 0 .
$$

From Lemma 1.4, one concludes that

$$
\lim _{n \rightarrow \infty}\left\|x_{n}-\lambda_{n}\right\|=0
$$

which implies that

$$
\lim _{n \rightarrow \infty}\left\|x_{n}-z_{n}\right\|=0
$$

Next, we show $x_{n}-y_{n} \rightarrow 0$ as $n \rightarrow \infty$. From (2.2), we have

$$
\begin{aligned}
\left\|\lambda_{n}-x\right\|^{2}= & \left\|\left(I-\mu \alpha_{n} F\right) z_{n}-\left(I-\mu \alpha_{n} F\right) x-\mu \alpha_{n} F x\right\|^{2} \\
\leq & \left\|\left(I-\mu \alpha_{n} F\right) z_{n}-\left(I-\mu \alpha_{n} F\right) x\right\|^{2}-2\left\langle\mu \alpha_{n} F x, \lambda_{n}-x\right\rangle \\
\leq & \left(1-\tau \alpha_{n}\right)\left\|z_{n}-x\right\|^{2}-2 \mu \alpha_{n}\left\langle F x, \lambda_{n}-x\right\rangle \\
\leq & \left(1-\tau \alpha_{n}\right)\left\|y_{n}-x\right\|^{2}-2 \mu \alpha_{n}\left\langle F x, \lambda_{n}-x\right\rangle \\
\leq & \left(1-\tau \alpha_{n}\right)\left\|x_{n}-x\right\|^{2}-\left(1-\tau \alpha_{n}\right) \gamma\left(1-\gamma\|A\|^{2}\right)\left\|\left(\operatorname{Res}_{s_{n}}^{N}-I\right) A x_{n}\right\|^{2} \\
& +2 \mu \alpha_{n}\|F x\|\left\|\lambda_{n}-x\right\| .
\end{aligned}
$$

Since squares of norms are convex, we have

$$
\begin{aligned}
\left\|x_{n+1}-x\right\|^{2} \leq & \left(1-\gamma_{n}\right)\left\|x_{n}-x\right\|^{2}+\gamma_{n}\left\|\lambda_{n}-x\right\|^{2} \\
\leq & \left(1-\gamma_{n} \tau \alpha_{n}\right)\left\|x_{n}-x\right\|^{2}-\gamma_{n}\left(1-\tau \alpha_{n}\right) \gamma\left(1-\gamma\|A\|^{2}\right)\left\|\left(\operatorname{Res}_{s_{n}}^{N}-I\right) A x_{n}\right\|^{2} \\
& +2 \gamma_{n} \mu \alpha_{n}\|F x\|\left\|\lambda_{n}-x\right\| .
\end{aligned}
$$

It follows that

$$
\begin{aligned}
& \gamma_{n}\left(1-\tau \alpha_{n}\right) \gamma\left(1-\gamma\|A\|^{2}\right)\left\|\left(\operatorname{Res}_{S_{n}}^{N}-I\right) A x_{n}\right\|^{2} \\
& \leq\left\|x_{n}-x\right\|^{2}-\left\|x_{n+1}-x\right\|^{2}+2 \gamma_{n} \mu \alpha_{n}\|F x\|\left\|\lambda_{n}-x\right\| \\
& \leq\left(\left\|x_{n}-x\right\|+\left\|x_{n+1}-x\right\|\right)\left\|x_{n}-x_{n+1}\right\|+2 \gamma_{n} \mu \alpha_{n}\|F x\|\left\|\lambda_{n}-x\right\| .
\end{aligned}
$$

From (2.8), one has

$$
\lim _{n \rightarrow \infty}\left\|x_{n}-x_{n+1}\right\|=0
$$


which together with (2.10) yields that

$$
\lim _{n \rightarrow \infty}\left\|A x_{n}-\operatorname{Res}_{s_{n}}^{N} A x_{n}\right\|=0 .
$$

Since $\operatorname{Res}_{s_{n}}^{N}$ is firmly nonexpansive, we obtain from inequality (1.3) that

$$
\begin{aligned}
&\left\|y_{n}-x\right\|^{2} \\
& \leq\left\langle x_{n}+\gamma A^{*}\left(\operatorname{Res}_{s_{n}}^{N}-I\right) A x_{n}-x, y_{n}-x\right\rangle \\
&= \frac{1}{2}\left\{\left\|x_{n}+\gamma A^{*}\left(\operatorname{Res}_{s_{n}}^{N}-I\right) A x_{n}-x\right\|^{2}+\left\|y_{n}-x\right\|^{2}-\left\|x_{n}+\gamma A^{*}\left(\operatorname{Res}_{s_{n}}^{N}-I\right) A x_{n}-y_{n}\right\|^{2}\right\} \\
&= \frac{1}{2}\left\{\gamma^{2}\left\|A^{*}\left(\operatorname{Res}_{r_{n}}^{N}-I\right) A x_{n}\right\|^{2}+2 \gamma\left\langle A^{*}\left(\operatorname{Res}_{s_{n}}^{N}-I\right) A x_{n}, x_{n}-x\right\rangle+\left\|x_{n}-x\right\|^{2}\right. \\
&\left.+\left\|y_{n}-x\right\|^{2}-\left\|x_{n}+\gamma A^{*}\left(\operatorname{Res}_{s_{n}}^{N}-I\right) A x_{n}-y_{n}\right\|^{2}\right\} \\
& \leq \frac{1}{2}\left\{\gamma^{2}\|A\|^{2}\left\|\left(\operatorname{Res}_{s_{n}}^{N}-I\right) A x_{n}\right\|^{2}+2 \gamma\left(\left\langle\left(\operatorname{Res}_{s_{n}}^{N}-I\right) A x_{n}, \operatorname{Res}_{s_{n}}^{N} A x_{n}-A x\right\rangle-\left\|\left(\operatorname{Res}_{s_{n}}^{N}-I\right) A x_{n}\right\|^{2}\right)\right. \\
&+\left\|x_{n}-x\right\|^{2}+\left\|y_{n}-x\right\|^{2}-\left\|x_{n}-y_{n}\right\|^{2}-2 \gamma\left\langle x_{n}-y_{n}, A^{*}\left(\operatorname{Res}_{s_{n}}^{N}-I\right) A x_{n}\right\rangle \\
&\left.\left.-\left\|\gamma A^{*}\left(\operatorname{Res}_{s_{n}}^{N}-I\right) A x_{n}\right\|^{2}\right)\right\} \\
& \leq \frac{1}{2}\left\{\left\|x_{n}-x\right\|^{2}+\left\|y_{n}-x\right\|^{2}+2 \gamma\|A\|\left\|x_{n}-y_{n}\right\|\left\|\left(\operatorname{Res}_{s_{n}}^{N}-I\right) A x_{n}\right\|-\left\|x_{n}-y_{n}\right\|^{2}\right\} .
\end{aligned}
$$

It follows that

$$
\left\|y_{n}-x\right\|^{2} \leq\left\|x_{n}-x\right\|^{2}+2 \gamma\|A\|\left\|x_{n}-y_{n}\right\|\left\|\left(\operatorname{Res}_{s_{n}}^{N}-I\right) A x_{n}\right\|-\left\|x_{n}-y_{n}\right\|^{2},
$$

which implies that

$$
\begin{aligned}
\left\|\lambda_{n}-x\right\|^{2} \leq & \left\|\left(I-\mu \alpha_{n} F\right) z_{n}-\left(I-\mu \alpha_{n} F\right) x\right\|^{2}-2\left\langle\mu \alpha_{n} F x, \lambda_{n}-x\right\rangle \\
\leq & \left(1-\tau \alpha_{n}\right)\left\|z_{n}-x\right\|^{2}-2 \mu \alpha_{n}\left\langle F x, \lambda_{n}-x\right\rangle \\
\leq & \left(1-\tau \alpha_{n}\right)\left\|x_{n}-x\right\|^{2}+2\left(1-\tau \alpha_{n}\right) \gamma\|A\|\left\|x_{n}-y_{n}\right\|\left\|\left(\operatorname{Res}_{s_{n}}^{N}-I\right) A x_{n}\right\| \\
& -\left(1-\tau \alpha_{n}\right)\left\|x_{n}-y_{n}\right\|^{2}-2 \mu \alpha_{n}\left\langle F x, \lambda_{n}-x\right\rangle .
\end{aligned}
$$

It follows that

$$
\begin{aligned}
\left\|x_{n+1}-x\right\|^{2} \leq & \left(1-\gamma_{n}\right)\left\|x_{n}-x\right\|^{2}+\gamma_{n}\left\|\lambda_{n}-x\right\|^{2} \\
\leq & \left(1-\gamma_{n}\right)\left\|x_{n}-x\right\|^{2}+\gamma_{n}\left(1-\tau \alpha_{n}\right)\left\|x_{n}-x\right\|^{2} \\
& +2 \gamma_{n}\left(1-\tau \alpha_{n}\right) \gamma\|A\|\left\|x_{n}-y_{n}\right\|\left\|\left(\operatorname{Res}_{s_{n}}^{N}-I\right) A x_{n}\right\| \\
& -\gamma_{n}\left(1-\tau \alpha_{n}\right)\left\|x_{n}-y_{n}\right\|^{2}-2 \gamma_{n} \mu \alpha_{n}\left\langle F x, \lambda_{n}-x\right\rangle \\
\leq & \left(1-\gamma_{n} \tau \alpha_{n}\right)\left\|x_{n}-x\right\|^{2}+2 \gamma_{n}\left(1-\tau \alpha_{n}\right) \gamma\|A\|\left\|x_{n}-y_{n}\right\|\left\|\left(\operatorname{Res}_{s_{n}}^{N}-I\right) A x_{n}\right\| \\
& -\gamma_{n}\left(1-\tau \alpha_{n}\right)\left\|x_{n}-y_{n}\right\|^{2}+2 \gamma_{n} \mu \alpha_{n}\|F x\|\left\|\lambda_{n}-x\right\| .
\end{aligned}
$$

This shows that

$$
\begin{aligned}
& \gamma_{n}\left(1-\tau \alpha_{n}\right)\left\|x_{n}-y_{n}\right\|^{2} \\
& \leq\left(1-\gamma_{n} \tau \alpha_{n}\right)\left\|x_{n}-x\right\|^{2}+2 \gamma_{n}\left(1-\tau \alpha_{n}\right) \gamma\|A\|\left\|x_{n}-y_{n}\right\|\left\|\left(\operatorname{Res}_{s_{n}}^{N}-I\right) A x_{n}\right\| \\
& \quad-\left\|x_{n+1}-x\right\|^{2}+2 \gamma_{n} \mu \alpha_{n}\|F x\|\left\|\lambda_{n}-x\right\| \\
& \leq\left(\left\|x_{n}-x\right\|+\left\|x_{n+1}-x\right\|\right)\left\|x_{n}-x_{n+1}\right\|+2 \gamma_{n} \gamma\|A\|\left\|x_{n}-y_{n}\right\|\left\|\left(\operatorname{Res}_{s_{n}}^{N}-I\right) A x_{n}\right\| \\
& \quad+2 \gamma_{n} \mu \alpha_{n}\|F x\|\left\|\lambda_{n}-x\right\| .
\end{aligned}
$$


So, we have $x_{n}-y_{n} \rightarrow 0$ as $n \rightarrow \infty$. Since $\left\{x_{n}\right\}$ is a bounded sequence, there exists a subsequence $\left\{x_{n_{i}}\right\}$ of $\left\{x_{n}\right\}$ such that $x_{n_{i}} \rightarrow x^{*}$ as $i \rightarrow \infty$. So, $y_{n_{i}} \rightarrow x^{*}, z_{n_{i}} \rightarrow x^{*}$ and $\lambda_{n_{i}} \rightarrow x^{*}$ as $k \rightarrow \infty$. Observe that

$$
y_{n}=\operatorname{Res}_{r_{n}}^{M}\left(x_{n}+\gamma A^{*}\left(\operatorname{Res}_{s_{n}}^{N}-I\right) A x_{n}\right),
$$

which implies from the maximal monotonicity of $M$ that

$$
\left\langle y_{n_{i}}-u, \frac{x_{n_{i}}-y_{n_{i}}+\gamma A^{*}\left(\operatorname{Res}_{s_{n_{i}}}^{N} A x_{n_{i}}-A x_{n_{i}}\right)}{\gamma}-v\right\rangle \geq 0,
$$

for $(u, v) \in M$. This shows that $0 \in M\left(x^{*}\right)$. Let $s$ be some positive real number. From Lemma 1.3, one has

It follows from (2.12) that

$$
\begin{aligned}
\left\|\operatorname{Res}_{s}^{N} A x_{n}-\operatorname{Res}_{s_{n}}^{N} A x_{n}\right\| & \leq\left\|A x_{n}-\frac{s}{s_{n}} A x_{n}-\left(1-\frac{s}{s_{n}}\right) \operatorname{Res}_{s_{n}}^{N} A x_{n}\right\| \\
& \leq \frac{\left|s_{n}-s\right|}{s}\left\|A x_{n}-\operatorname{Res}_{s_{n}}^{N} A x_{n}\right\| .
\end{aligned}
$$

Taking into the fact that

$$
\lim _{n \rightarrow \infty}\left\|\operatorname{Res}_{s_{n}}^{N} A x_{n}-\operatorname{Res}_{s}^{N} A x_{n}\right\|=0 .
$$

$$
\left\|\operatorname{Res}_{s}^{N} A x_{n}-A x_{n}\right\| \leq\left\|\operatorname{Res}_{s_{n}}^{N} A x_{n}-A x_{n}\right\|+\left\|\operatorname{Res}_{s}^{N} A x_{n}-\operatorname{Res}_{s_{n}}^{N} A x_{n}\right\|,
$$

we conclude that $\lim _{n \rightarrow \infty}\left\|A x_{n}-\operatorname{Res}_{s}^{N} A x_{n}\right\|=0$. We also have

$$
\lim _{i \rightarrow \infty}\left\|A x_{n_{i}}-\operatorname{Res}_{s}^{N} A x_{n_{i}}\right\|=0 .
$$

From Lemma 1.1, we find that $A x^{*} \in F i x\left(\operatorname{Res}_{s}^{N}\right)$, that is, $0 \in N\left(A x^{*}\right)$.

Next, we prove that $x^{*}$ is also a fixed point of $T$. From (2.1), we have

$$
T y_{n}-y_{n}=\frac{z_{n}-y_{n}}{1-\beta_{n}} \text {. }
$$

Since $\left\{\beta_{n}\right\}$ is bounded away from 1 , we find that $\lim _{n \rightarrow \infty}\left\|T y_{n}-y_{n}\right\|=0$. Since $I-T$ is demiclosed, we obtain that $x^{*} \in \operatorname{Fix}(T)$.

Since $F$ is strongly monotone and Lipschitz continuous, we next use $\bar{x}$ to denote the unique solution of variational inequality (2.1). That is,

$$
\limsup _{n \rightarrow \infty}\left\langle F(\bar{x}), \bar{x}-\lambda_{n}\right\rangle \leq 0 .
$$

We have

$$
\begin{aligned}
\left\|\lambda_{n}-\bar{x}\right\|^{2} & =\left\|\left(I-\mu \alpha_{n} F\right) z_{n}-\left(I-\mu \alpha_{n} F\right) \bar{x}-\mu \alpha_{n} F \bar{x}\right\|^{2} \\
& \leq\left\|\left(I-\mu \alpha_{n} F\right) z_{n}-\left(I-\mu \alpha_{n} F\right) \bar{x}\right\|^{2}-2 \mu \alpha_{n}\left\langle F \bar{x}, \lambda_{n}-\bar{x}\right\rangle \\
& \leq\left(1-\tau \alpha_{n}\right)^{2}\left\|z_{n}-\bar{x}\right\|^{2}-2 \mu \alpha_{n}\left\langle F \bar{x}, \lambda_{n}-\bar{x}\right\rangle \\
& \leq\left(1-\tau \alpha_{n}\right)^{2}\left\|y_{n}-\bar{x}\right\|^{2}-2 \mu \alpha_{n}\left\langle F \bar{x}, \lambda_{n}-\bar{x}\right\rangle \\
& \leq\left(1-\tau \alpha_{n}\right)^{2}\left\|x_{n}-\bar{x}\right\|^{2}+2 \mu \alpha_{n}\left\langle F \bar{x}, \bar{x}-\lambda_{n}\right\rangle,
\end{aligned}
$$

which implies that

$$
\begin{aligned}
\left\|x_{n+1}-\bar{x}\right\|^{2} & \leq\left(1-\gamma_{n}\right)\left\|x_{n}-\bar{x}\right\|^{2}+\gamma_{n}\left\|\lambda_{n}-\bar{x}\right\|^{2} \\
& \leq\left(1-\gamma_{n}\right)\left\|x_{n}-\bar{x}\right\|^{2}+\gamma_{n}\left(1-\tau \alpha_{n}\right)^{2}\left\|x_{n}-\bar{x}\right\|^{2}+2 \gamma_{n} \mu \alpha_{n}\left\langle F \bar{x}, \bar{x}-\lambda_{n}\right\rangle \\
& \leq\left(1-\xi_{n}\right)\left\|x_{n}-\bar{x}\right\|^{2}+\xi_{n} \frac{\tau^{2} \alpha_{n}\left\|x_{n}-\bar{x}\right\|^{2}+2 \mu\left\langle F \bar{x}, \bar{x}-\lambda_{n}\right\rangle}{2 \tau}
\end{aligned}
$$


where $\xi_{n}=2 \tau \gamma_{n} \alpha_{n}$. Observe $\xi_{n} \rightarrow 0, \sum_{n=1}^{\infty} \xi_{n}=\infty$ and

$$
\limsup _{n \rightarrow \infty} \frac{\tau^{2} \alpha_{n}\left\|x_{n}-\bar{x}\right\|^{2}+2 \mu\left\langle F \bar{x}, \bar{x}-\lambda_{n}\right\rangle}{2 \tau} \leq 0 .
$$

Using Lemma 1.5, we obtain that $\left\|x_{n}-\bar{x}\right\| \rightarrow 0$. This completes the proof.

From Theorem 2.1, the following result involving nonexpansive mappings are not hard to derived easily.

Corollary 2.1. Let $H_{1}$ and $H_{2}$ be two real Hilbert spaces. Let $T$ be a nonexpansive mapping on $H_{1}$ with fixed points. Let $A: H_{1} \rightarrow H_{2}$ be a bounded linear operator and let $A^{*}$ be the adjoint operator of $A$. Let $F: H_{1} \rightarrow H_{1}$ be a $\mathscr{L}$-Lipschitz continuous and $\tau$-strongly monotone mapping. Let $M$ be a maximal monotone mapping on $\mathrm{H}_{1}$ and let $\mathrm{N}$ be a maximal monotone mapping on $\mathrm{H}_{2}$. Assume that $\operatorname{SFP}(M, N) \cap \operatorname{Fix}(T) \neq \emptyset$. Let $\left\{x_{n}\right\}$ be a sequence generated by the following process:

$$
\left\{\begin{array}{l}
x_{1} \in H_{1}, \\
z_{n}=\operatorname{TRes}_{r_{n}}^{M}\left(x_{n}+\gamma A^{*}\left(\operatorname{Res}_{s_{n}}^{N}-I\right) A x_{n}\right), \\
x_{n+1}=\left(1-\gamma_{n}\right) x_{n}+\gamma_{n}\left(I-\mu \alpha_{n} F\right) z_{n}, \quad n \geq 1,
\end{array}\right.
$$

where $\left\{r_{n}\right\}$ and $\left\{s_{n}\right\}$ are two positive real number sequences, $\left\{\alpha_{n}\right\}$, and $\left\{\gamma_{n}\right\}$ are sequences in $(0,1)$, $\mu$ and $\gamma$ are two positive real numbers, and $\left\{r_{n}\right\}$ is a positive sequence. Assume that $\lim _{n \rightarrow \infty} \alpha_{n}=0$, $\sum_{n=1}^{\infty} \alpha_{n}=\infty,\left\{\gamma_{n}\right\}$ is in $\left[\rho, \rho^{\prime}\right]$, where $\rho$ and $\rho^{\prime}$ are two real numbers in $(0,1)$, such that $\lim _{n \rightarrow \infty} \mid \gamma_{n+1}-$ $\gamma_{n}\left|=0, \liminf _{n \rightarrow \infty} r_{n}>0, \lim _{n \rightarrow \infty}\right| r_{n+1}-r_{n}\left|<\infty, \liminf _{n \rightarrow \infty} s_{n}>0, \lim _{n \rightarrow \infty}\right| s_{n+1}-s_{n} \mid<\infty, \mu \in\left(0, \frac{2 \tau}{\mathscr{L}^{2}}\right)$, and $\gamma \in\left(0, \frac{1}{\|A\|^{2}}\right)$. Then $\left\{x_{n}\right\}$ converge strongly to $\bar{x} \in \operatorname{SFP}(M, N) \cap F i x(T)$, which is the unique solution of the following variational inequality

$$
\langle\bar{x}-y, F \bar{x}\rangle \leq 0, \quad \forall y \in \operatorname{SFP}(M, N) \cap F i x(T) .
$$

Proof. Every nonexpansive mapping is a strict pseudocontraction with coefficient 0 . Setting $\beta_{n}=0$, we have the desired conclusion immediately.

From Theorem 2.1, we also have the following result on split inclusion problem (1.1)-(1.2).

Corollary 2.2. Let $H_{1}$ and $H_{2}$ be two real Hilbert spaces. Let $A: H_{1} \rightarrow H_{2}$ be a bounded linear operator and let $A^{*}$ be the adjoint operator of $A$. Let $F: H_{1} \rightarrow H_{1}$ be a $\mathscr{L}$-Lipschitz continuous and $\tau$-strongly monotone mapping. Let $M$ be a maximal monotone mapping on $H_{1}$ and let $N$ be a maximal monotone mapping on $\mathrm{H}_{2}$. Assume that $\operatorname{SFP}(M, N) \neq \emptyset$. Let $\left\{x_{n}\right\}$ be a sequence generated by the following process:

$$
\left\{\begin{array}{l}
x_{1} \in H_{1}, \\
z_{n}=\operatorname{Res}_{r_{n}}^{M}\left(x_{n}+\gamma A^{*}\left(\operatorname{Res}_{s_{n}}^{N}-I\right) A x_{n}\right), \\
x_{n+1}=\left(1-\gamma_{n}\right) x_{n}+\gamma_{n}\left(I-\mu \alpha_{n} F\right) z_{n}, \quad n \geq 1,
\end{array}\right.
$$

where $\left\{r_{n}\right\}$ and $\left\{s_{n}\right\}$ are two positive real number sequences, $\left\{\alpha_{n}\right\}$, and $\left\{\gamma_{n}\right\}$ are sequences in $(0,1)$, $\mu$ and $\gamma$ are two positive real numbers, and $\left\{r_{n}\right\}$ is a positive sequence. Assume that $\lim _{n \rightarrow \infty} \alpha_{n}=0$, $\sum_{n=1}^{\infty} \alpha_{n}=\infty,\left\{\gamma_{n}\right\}$ is in $\left[\rho, \rho^{\prime}\right]$, where $\rho$ and $\rho^{\prime}$ are two real numbers in $(0,1)$, such that $\lim _{n \rightarrow \infty} \mid \gamma_{n+1}-$ $\gamma_{n}\left|=0, \liminf _{n \rightarrow \infty} r_{n}>0, \lim _{n \rightarrow \infty}\right| r_{n+1}-r_{n}\left|<\infty, \liminf _{n \rightarrow \infty} s_{n}>0, \lim _{n \rightarrow \infty}\right| s_{n+1}-s_{n} \mid<\infty, \mu \in\left(0, \frac{2 \tau}{\mathscr{L}^{2}}\right)$, and $\gamma \in\left(0, \frac{1}{\|A\|^{2}}\right)$. Then $\left\{x_{n}\right\}$ converge strongly to $\bar{x} \in \operatorname{SFP}(M, N)$, which is the unique solution of the following variational inequality $\langle\bar{x}-y, F \bar{x}\rangle \leq 0, \forall y \in \operatorname{SFP}(M, N)$. 


\section{Acknowledgments}

The author is grateful to the referee for useful suggestions which improved the contents of this paper. This paper was supported by the National Natural Science Foundation of China under Grant 11601348.

\section{REFERENCES}

[1] A. Moudafi, On the difference of two maximal monotone operators: Regularization and algorithmic approaches, Appl. Math. Comput. 202 (2008), 446-452.

[2] S.Y. Cho, X. Qin, L. Wang, Strong convergence of a splitting algorithm for treating monotone operators, Fixed Point Theory Appl. 2014 (2014), Article ID 94.

[3] B.A. Bin Dehaish, et al., A regularization projection algorithm for various problems with nonlinear mappings in Hilbert spaces, J. Inequal. Appl. 2015 (2015), Article ID 51.

[4] B.A. Bin Dehaish, et al., Weak and strong convergence of algorithms for the sum of two accretive operators with applications, J. Nonlinear Convex Inequal. Appl. 16 (2015), 1321-1336.

[5] S.S. Chang, C.F. Wen and J.C. Yao, Common zero point for a finite family of inclusion problems of accretive mappings in Banach spaces, Optimization, 67 (2018), 1183-1196.

[6] X. Qin, J.C. Yao, Weak convergence of a Mann-like algorithm for nonexpansive and accretive operators, J. Inequal. Appl. 2016 (2016), Articl ID 232.

[7] X. Qin, S.Y. Cho, L. Wang, Iterative algorithms with errors for zero points of m-accretive operators, Fixed Point Theory Appl. 2013 (2013), Article ID 148.

[8] Y. Censor, T. Elfving, A multiprojection algorithm using Bregman projections in a product space, Numer. Algorightm 8 (1994), 221-239.

[9] Y. Censor, T. Bortfeld, N. Martin, A. Trofimov, A unified approach for inversion problem in intensity-modulated radiation therapy, Phys. Med. Biol. 51 (2006), 2353-2365.

[10] Y. Censor, T. Elfving, N. Kopf, T. Bortfeld, The multiple-sets split feasiblility problem and its applications, Inverse Probl. 21 (2005) 2071-2084.

[11] Y. Censor, A. Motova, A. Segal, Perturbed projections ans subgradient projiections for the multiple-sets split feasibility problem, J. Math. Anal. Appl. 327 (2007), 1244-1256.

[12] N. Fang, Some results on split variational inclusion and fixed point problems in Hilbert spaces, Commun. Optim. Theory 2017 (2017), Article ID 5.

[13] X. Qin, A. Petrusel, J.C. Yao, CQ iterative algorithms for fixed points of nonexpansive mappings and split feasibility problems in Hilbert spaces, J. Nonlinear Convex Anal. 19 (2018), 157-165.

[14] Y. Yang, Q. Yuan, A hybrid descent iterative algorithm for a split inclusion problem, J. Nonlinear Funct. Anal. 2018 (2018), Article ID 42.

[15] P. Majee, C. Nahak, On inertial proximal algorithm for split variational inclusion problems, Optimization 67 (2018), 1701-1716.

[16] F.E. Browder, W.V. Petryshyn, Construction of fixed points of nonlinear mappings in Hilbert space, J. Math. Anal. Appl. 20 (1967), 197-228.

[17] G. Cai, Y. Shehu, O.S. Iyiola, Strong convergence theorems for fixed point problems for strict pseudo-contractions and variational inequalities for inverse-strongly accretive mappings in uniformly smooth Banach spaces, J. Fixed Point Theory Appl. 21 (2019), Article ID 41.

[18] P.N. Anh, N.X. Phuong, Fixed point methods for pseudomonotone variational inequalities involving strict pseudocontractions, 64 (2015), 1841-1854.

[19] N.T. Vinh, P.T. Hoai, Some subgradient extragradient type algorithms for solving split feasibility and fixed point problems, Math. Meth. Appl. Sci. 39 (2016), 3808-3823.

[20] Y. Yamada, The hybrid steepest-descent method for variational inequalities problems over the intersection of the fixed point sets of nonexpansive mappings, In: Butnariu, D., Censor, Y., Reich, S. (eds.) Inherently Parallel Algorithms in Feasibility and Optimization and Their Applications, pp. 473-504. North-Holland, Amsterdam, 2001.

[21] V. Barbu, Nonlinear Semigroups and Differential Equations in Banach Spaces, Noordhoff, Amsterdam, 1976. 
[22] T. Suzuki, Strong convergence theorems for infinite families of nonexpansive mappings in general Banach spaces, Fixed Point Theory Appl. 2005 (2005), Article ID 685918.

[23] Z. Xue, H. Zhou, Y.J. Cho, Iterative solutions of nonlinear equations for m-accretive operators in Banach spaces, J. Nonlinear Convex Anal. 1 (2000), 313-320. 